\title{
INSULIN-LIKE GROWTH FACTOR BINDING PROTEIN (IGFBP6) IS A CROSS-SPECIES TENDON MARKER
}

\author{
A.J. Turlo*, A.J. Mueller-Breckenridge, D.E. Zamboulis, S.R. Tew, E.G. Canty-Laird and P.D. Clegg
}

Musculoskeletal Biology I, Institute of Ageing and Chronic Disease, University of Liverpool, Liverpool, UK

\begin{abstract}
The main challenge in tendon injury management is suboptimal tissue healing that fails to re-establish original tendon function. Tissue bioengineering is a promising approach for tendon therapy, with potential to improve its functional outcomes. However, evaluation criteria for tissue-engineered tendon are unclear due to the lack of specific markers of differentiated tendon. The study aim was to identify a panel of genes that characterised tendons in comparison to cartilage or muscles and validate those genes, both in human and key species used as models for tendon diseases. Gene expression profiling of rat tendon and cartilage in whole-tissue samples and primary tenocytes and chondrocytes was undertaken using two independent microarray platforms. Genes that demonstrated high expression correlation across two assays were validated by qRT-PCR in rat tendon relative to cartilage and muscle. Five genes demonstrating the highest tendonrelated expression in the validation experiment ( $A S P N, E C M 1$, IGFBP6, TNMD, THBS4) were further evaluated by qRT-PCR in ovine, equine and human tissue. The group of tendon markers, identified by unbiased transcriptomic analysis of rat musculoskeletal tissues, demonstrated species-dependent profiles of expression. Insulin-like growth factor binding protein 6 (IGFBP6) was identified as the only universal tendon marker. Further investigation in equine tendon showed that IGFBP6 expression was not affected by ageing or tendon function but decreased in anatomical regions subjected to elevated compressive force. IGFBP6 is a robust cross-species marker of tendon phenotype and may find application in evaluation of tendon physiology and guided differentiation of permissive cells towards functional tenocytes.
\end{abstract}

Keywords: Bioengineering, tissue engineering, tendonitis, biomarker, translational biology.

*Address for correspondence: Agnieszka J. Turlo, Institute of Ageing and Chronic Disease, William Henry Duncan Building, 6 West Derby Street, L7 8TX Liverpool, UK.

Telephone number: +4407874083774 Email: a.turlo@liverpool.ac.uk

Copyright policy: This article is distributed in accordance with Creative Commons Attribution Licence (http://creativecommons.org/licenses/by-sa/4.0/).

\section{Introduction}

The tendon is a highly specialised connective tissue that transmits the mechanical force of muscle contraction to the bone. Tendon histological structure, dominated by dense extracellular matrix (ECM) with sparsely distributed resident cells, reflects adaptation to high physical demands of tensile strength resistance (Thorpe and Screen, 2016). Despite their small contribution to the overall tissue mass, tendon cells (tenocytes) are primarily responsible for its mechano-physical properties, through synthesising the bulk of ECM mass during development and modifying its composition according to the prevailing biomechanical stimuli (Kjær et al., 2009; Testa et al., 2017; Zhang et al., 2010). Aberrant cell response to injury is thought to be one of the causes underlying the development of chronic tendinopathies and is characterised by changes in ECM organisation which are inconsistent with optimal tendon function (Titan and Andarawis-Puri, 2016). Alterations in resident cell phenotype, primarily chondrogenic differentiation, are widely identified in tendinopathies in association with degenerate tendon ECM (Asai et al., 2014; Burssens et al., 2013; Clegg et al., 2007; Titan and Andarawis-Puri, 2016). Therefore, promoting functional tendon tissue repair at the injury site and directing appropriate cell response subsequent to injury are key goals for tendinopathy treatment.

Tendon bioengineering involves the application of tenocytes as well as mesenchymal stem cells (MSCs) to develop engineered tendon that may be subsequently used as grafts for tendon injury repair (Müller et al., 2013). This promising therapeutic approach is challenged by the tendency of tendon cells to undergo phenotypic transitions when maintained under cell-culture conditions (Mueller et al., 2016; Taylor et al., 2009; Yao et al., 2006). Tenocyte- and stem-cell- 
derived constructs developed in vitro are at risk of cell dedifferentiation and aberrant differentiation towards chondrogenic and osteogenic lineages, possibly due to the common mesenchymal origin of musculoskeletal tissues (Harris et al., 2004; Tan et al., 2012). In order to evaluate outcomes of tendon tissueengineering and further optimise culture conditions promoting teno-lineage differentiation, molecular markers of a functional adult tendon need to be defined. Genes currently investigated in tenocyte phenotype characterisation, such as scleraxis (SCX), tenomodulin (TNMD), tenascin $C(T N C)$ and type 1 collagen $\alpha 1$ chain (COL1A1), are often primarily derived from developmental studies and their validity as markers of mature tenocytes can vary depending on tendon type, anatomical region, species and age (Howell et al., 2017; Jelinsky et al., 2010; Kuemmerle et al., 2016; Mehr et al., 2000; Murchison et al., 2007; Peffers et al., 2015; Taylor et al., 2009). Moreover, their expression can also be maintained in tendons undergoing pathological transformation such as fibrosis, cartilage formation or endochondral ossification (Agarwal et al., 2017; Howell et al., 2017; Mehr et al., 2000) further questioning their utility as markers of normal tendon phenotype. Several studies utilising unbiased approaches to identify novel tendon-selective markers by comparative expression profiling of various musculoskeletal tissues have been reported (Jelinsky et al., 2010; Kuemmerle et al., 2016). However, resilience of these new targets to the above described variables has yet to be defined, limiting their application to the species, tendon type and region they were derived from.

Rat, sheep and horse are frequently used animal models for tendon research (Lui et al., 2011). Rat Achilles tendon shows anatomical complexity similar to that of humans (Szaro et al., 2012) and are suitable for manipulation methods which are impractical in larger species (Andarawis-Puri and Flatow, 2011; Brunton et al., 2018). The size of sheep and equine flexor tendons makes them more suitable for investigating the efficacy of clinical interventions in experimentally induced tendon lesions (Dahlgren et al., 2002; Hausmann et al., 2009; Song et al., 2010). Additionally, horse and sheep tendons exhibit a hierarchical microstructure analogous to humans, with two distinct anatomical compartments: collagenrich fascicles and looser interfascicular matrix (IFM). The fascicle-IFM interaction is considered to play a major role in tendon mechanical behaviour (Thorpe et al., 2015); therefore, conservation of that microstructure is a highly desirable feature in a relevant animal model. The horse is of particular interest for tendon research due to the natural occurrence of overuse tendinopathy and age-related degeneration (Patterson-Kane et al., 2012; Peffers et al., 2014), which are also the main disorders affecting tendon health in humans (Scott et al., 2015).

The present study aimed at identifying novel gene markers that exhibited clearly higher expression in adult tendon relative to cartilage and muscle and validated them across the key model species, rat, sheep and horse, as well as human tendons.

\section{Materials and Methods}

\section{Tissue collection}

Tissue collection from different species was individually approved by the University of Liverpool Institute of Veterinary Sciences Research Ethics Committee. Details of sample origin and donor demographics are presented in Table 1. Tissues from rat were collected under the Schedule I of the UK Animal Scientific Procedures Act (1986). Equine samples were collected as a by-product of the agricultural industry. Sheep tissues were collected as part of routine post-mortem examinations for Fasciola hepatica at the School of Veterinary Science, University of Liverpool. Human cadaveric Achilles tendon samples were obtained following ethical approval by North East Tyne and Wear South Research Ethics Committee (14/NE/0154). Human cadaveric femorotibial joint cartilage was obtained from a commercial biorepository (ProteoGenex, Inglewood, CA, USA) and provided with proof of donor consent approval by the local ethical authority at the sample collection site. Human muscle samples were represented by primary myoblasts isolated from muscle biopsy material collected intraoperatively from patients undergoing surgical treatment for hallux valgus, with patient consent and approval from the University of Liverpool, University Hospital Aintree Hospital and South West Wales Research Ethics Committee (13/WA/0374). Rat, sheep and equine whole tissue specimens were dissected and stored immediately upon collection in RNAlater ${ }^{\mathrm{TM}}$ Stabilisation Solution (Invitrogen) according to the manufacturer's protocol $\left(24 \mathrm{~h}\right.$ at $4{ }^{\circ} \mathrm{C}$ followed by long-term storage at $-80{ }^{\circ} \mathrm{C}$ ). According to the information provided by the biobank, human cadaveric tendon and cartilage samples were preserved by flash-freezing within 30 to $60 \mathrm{~min}$ of excision in liquid nitrogen and stored at $-80^{\circ} \mathrm{C}$ prior to RNA isolation.

\section{Histology}

To evaluate phenotype of different regions of equine SDFT, samples of SDFT midportion and sesamoid region were fixed in $10 \%$ buffered formalin for 48 $72 \mathrm{~h}$, dehydrated and embedded in paraffin-wax on their longitudinal axis. Then, the tissue blocks were cut into $5-\mu \mathrm{m}$-thick sections, collected on polylysine slides and stained with haematoxylin and eosin (H\&E).

\section{Cell isolation}

Human

Human primary myoblast isolation was undertaken according to a previously published method (Soriano-Arroquia et al., 2017). Briefly, samples were washed in Dulbecco's phosphate-buffered saline 
Table 1. Detailed description of samples used for respective experiments. Information on donor sex in equine samples was not available.

\begin{tabular}{|c|c|c|c|}
\hline \multicolumn{4}{|c|}{ Microarray } \\
\hline \multirow[b]{2}{*}{ Rat, F344 } & \multirow[b]{2}{*}{12 weeks; male } & \multirow[b]{2}{*}{$n=5$} & Tendon (AT, DDFT, tail tendon) \\
\hline & & & $\begin{array}{l}\text { Cartilage (coxo-femoral and } \\
\text { femorotibial joint) }\end{array}$ \\
\hline \multirow[b]{2}{*}{ Rat, Lewis } & \multirow[b]{2}{*}{12 weeks; male } & \multirow[b]{2}{*}{$n=4$} & Primary tenocytes (AT, DDFT ) \\
\hline & & & $\begin{array}{l}\text { Primary chondrocytes (coxo-femoral } \\
\text { and femorotibial joint) }\end{array}$ \\
\hline \multicolumn{4}{|c|}{ RT-qPCR cross-species validation } \\
\hline \multirow{3}{*}{ Rat, Lewis } & \multirow{3}{*}{12 weeks; male } & \multirow{3}{*}{$n=6$} & Tendon (AT, DDFT) \\
\hline & & & $\begin{array}{c}\text { Cartilage (coxo-femoral and } \\
\text { femorotibial joint) }\end{array}$ \\
\hline & & & Muscle (quadriceps femoris) \\
\hline \multirow{3}{*}{ Sheep } & \multirow{3}{*}{ Adult female } & \multirow{3}{*}{$n=6$} & Tendon (DDFT) \\
\hline & & & Cartilage (metatarsophalangeal joint) \\
\hline & & & Muscle (quadriceps femoris) \\
\hline \multirow{3}{*}{ Horse } & $9-14$ years $(11.9 \pm 2.2$ years $)$ & $n=8$ & Tendon (SDFT midportion) \\
\hline & $4-15$ years $(8.4 \pm 6$ years $)$ & $n=5$ & Cartilage (metatarsophalangeal joint) \\
\hline & & $n=4$ & Muscle (trapezius) \\
\hline \multirow{3}{*}{ Human } & $\begin{array}{c}56-93 \text { years ( } 82.4 \pm 15.4 \text { years); } \\
3 \text { female, } 2 \text { male }\end{array}$ & $n=5$ & Tendon (AT midportion) \\
\hline & $\begin{array}{c}32-75 \text { years ( } 56 \pm 20.8 \text { years }) \\
\text { male }\end{array}$ & $n=6$ & Cartilage (femorotibial joint) \\
\hline & $\begin{array}{l}31-38 \text { years }(32.2 \pm 2.7 \text { years }) \\
\text { female }\end{array}$ & $n=6$ & $\begin{array}{c}\text { Primary myoblasts } \\
\text { (extensor digitorum brevis, tibialis anterior, } \\
\text { abductor halluces) }\end{array}$ \\
\hline \multicolumn{4}{|c|}{ RT-qPCR IGFBP6 in tendon ageing and function } \\
\hline \multirow{11}{*}{ Horse } & $0 \mathrm{~d}$ & $n=4$ & \multirow{7}{*}{ SDFT midportion } \\
\hline & $0-1$ months & $n=3$ & \\
\hline & 3-6 months & $n=4$ & \\
\hline & 12-24 months & $n=4$ & \\
\hline & $3-5$ years & $n=5$ & \\
\hline & 9-11 years & $n=5$ & \\
\hline & 18-22 years & $n=4$ & \\
\hline & \multirow{2}{*}{ 3-5 years } & \multirow{2}{*}{$n=5$} & SDFT midportion \\
\hline & & & CDET \\
\hline & \multirow{2}{*}{ 5-7 years } & \multirow{2}{*}{$n=5$} & SDFT midportion \\
\hline & & & SDFT sesamoid region \\
\hline
\end{tabular}

and dissected in a sterile Petri dish in warm $\left(37^{\circ} \mathrm{C}\right)$ collagenase-dispase- $\mathrm{CaCl}_{2}$ solution $[1.5 \mathrm{U} / \mathrm{mL}$ of collagenase D, $2.4 \mathrm{U} / \mathrm{mL}$ of Dispase II and $2.5 \mathrm{mM}$ $\mathrm{CaCl}_{2}$ in serum-free Dulbecco's modified eagle medium (DMEM)] followed by a 40 min incubation in $50 \mathrm{~mL}$ Falcon tube. Digestion was stopped by adding sterile growth medium $(4 \mathrm{~mL}$ of DMEM containing $20 \%$ foetal bovine serum, $1 \%$ L-glutamine and $1 \%$ penicillin-streptomycin) and the sample was filtered through a $70 \mu \mathrm{m}$ cell strainer. Cells were pelleted by centrifugation at $443 \times g$ for $5 \mathrm{~min}$ at room temperature and stored in TriReagent (Sigma-Aldrich) at $-80^{\circ} \mathrm{C}$ for RNA isolation.

\section{Rat}

In addition to whole tissue samples, expression profiling of rat primary tenocytes and chondrocytes was undertaken. Primary tenocytes were isolated from Achilles tendon (AT) and deep digital flexor tendon (DDFT), chondrocytes from femorotibial and coxo-femoral joint articular cartilage; both were pooled for each individual. Tissue samples were minced and washed twice in serum-free DMEM, containing penicillin $(100 \mathrm{U} / \mathrm{mL})$, streptomycin $(100 \mu \mathrm{g} / \mathrm{mL})$ and amphotericin B $(2 \mu \mathrm{g} / \mathrm{mL})$. Cells were isolated by collagenase digestion $(0.4 \%$, type 2 , Worthington) for over $20 \mathrm{~h}$ at $37^{\circ} \mathrm{C}$. Next, samples were centrifuged: cartilage for $8 \mathrm{~min}$ at $500 \times g$ and tendon for $15 \mathrm{~min}$ at $1,000 \times g$, to ensure that partially digested fascicular material would be spinned-down (Ritty et al., 2003). Supernatant was removed and samples incubated for $4 \mathrm{~h}$ at $37^{\circ} \mathrm{C}$ in $0.25 \%$ bovine pancreas trypsin (Sigma-Aldrich) in serum-free DMEM. Samples were centrifuged as 
before, washed with complete medium, re-suspended and filtered through a $70 \mu \mathrm{m}$ sterile cell strainer. Cells were pelleted by centrifugation and cell pellets stored in TriReagent.

\section{RNA extraction}

Whole tissue samples were pulverised under liquid nitrogen. Ground-up tissue and cell pellets were incubated for $10 \mathrm{~min}$ at room temperature in TriReagent. RNA was extracted using an acid guanidinium thiocyanate-phenol-chloroform method (Chomczynski and Sacchi, 2006). Following RNA resuspension in $75 \%$ ethanol, DNAse digestion and purification were performed using RNeasy Mini Kit (QIAGEN). RNA was quantified using an ND1000 spectrophotometer (Thermo Fischer Scientific). For the microarray experiment, RNA integrity was measured using a Bioanalyser (Agilent), with all samples obtaining RNA integrity number (RIN) scores $>8$. In samples used for quantitative real-time reverse transcription polymerase chain reaction (qRT-PCR), RNA purity was assessed based on the $A_{260} / A_{280}$ absorbance ratio and samples with ratio $<1.8$ were not accepted for cDNA preparation. RNA quantity used for the reverse transcription was normalised across different tissue types within each species for cross-tissue comparison of gene expression.

\section{Rat microarray \\ Samples}

Gene expression profiling of rat tendon was undertaken using two independent microarray platforms: i) Illumina RatRef v12 platform (ArrayExpress accession code: E-MTAB-4800) as previously described (Mueller et al., 2016) and ii) Affymetrix GeneST Rat platform. Sample preparation for the two platforms differed as follows: i) RNA was extracted from whole tendon and cartilage from 12-week-old male F344 rats $(n=5)$ for profiling by Illumina microarray; ii) RNA extraction for Affymetrix profiling was derived from primary tenocytes and chondrocytes isolated as described above from tissues obtained from 12-week-old male Lewis rats $(n=4)$. Sample preparation for each platform followed the standard protocol recommended by each manufacturer. Illumina microarray profiling was performed by The Genome Centre, Queen Mary, University of London, UK. Affymetrix microarray profiling was performed by Tepnel Pharma Services, Hologic Ltd., Manchester, UK.

\section{Data analysis}

Raw gene expression data from both platforms was pre-processed and interrogated using software packages implemented in $R$ (Web ref. 1). Expression data were $\log _{2}$-transformed and normalised (loess) across all samples. Differential expression analysis was performed using methods previously described (Smyth, 2005). Pearson's correlation coefficient was calculated for genes differentially expressed in tendon in both platforms.

\section{Quantitative real-time PCR \\ Samples}

Candidate marker genes identified in the rat microarray were further validated in different musculoskeletal tissues of human and key model species by qRT-PCR. A summary of sample information is presented in Table 1. Briefly, tissue samples included: i) whole tendon, cartilage and muscle from rat, sheep and horse; ii) human whole tendon, cartilage and isolated primary myoblasts.

Insulin-like growth factor binding protein 6 (IGFBP6) expression was further analysed in equine superficial digital flexor tendon (SDFT) in relation to age and anatomical location. RNA extracted from the SDFT midportion was analysed across different age groups, from full formed foetuses to old horses (Table 1). To investigate the impact of anatomical differences on IGFBP6 expression in tendons, the SDFT midportion was compared to the SDFT sesamoid region (compressed by proximal sesamoid bones) in young mature horses. Additionally, expression in energy-storing SDFT and positional common digital extensor tendon (CDET) was compared to determine the relation between tendon function and IGFBP 6 expression.

\section{Primers}

Primers were designed using NCBI PrimerBlast (Ye et al., 2012) against the most recent published record (04/2016). All primers were species-specific and amplification efficiency was calculated using tendon cDNA in six 10-fold serial dilutions. Primer efficiencies were used to correct normalised cycle threshold $\left(C_{t}\right)$ values (Pfaffl, 2006). All primers were sourced from Eurogentec S.A. (Liege, Belgium) except for equine TNMD, which was custom designed by PrimerDesign (UK), and human IGFBP6 and TNMD, which were provided by Bio-Rad. The sequences of custom-designed primers are provided in Table 2.

\section{cDNA production and $q R T-P C R$}

Reverse transcription was performed using M-MLV reverse transcriptase and random-hexamer oligonucleotides (all Promega). Quantitative PCR (qPCR) was performed on a LightCycler 480 II (Roche) using 96-well plates (STARLAB). Negative controls (cDNA) were included for each primer test. A proprietary qPCR SYBR ${ }^{\mathrm{TM}}$ master mix (Takyon $^{\mathrm{TM}}$, no ROX passive reference dye, deoxythymidine triphosphate (dTTP); Eurogentec S.A.) was used for all analyses apart from human IGFBP6 and TNMD where Sso Advanced ${ }^{\mathrm{TM}}$ master mix by Bio-Rad was used. The manufacturer's recommended protocol for PCR was used: briefly, enzyme activation ( $3 \mathrm{~min}, 95^{\circ} \mathrm{C}$ ) followed by 40 cycles consisting of denaturation $\left(3 \mathrm{~s}, 95^{\circ} \mathrm{C}\right)$ and annealing-extension $\left(20 \mathrm{~s}, 60^{\circ} \mathrm{C}\right)$. Products of qPCR amplification were 


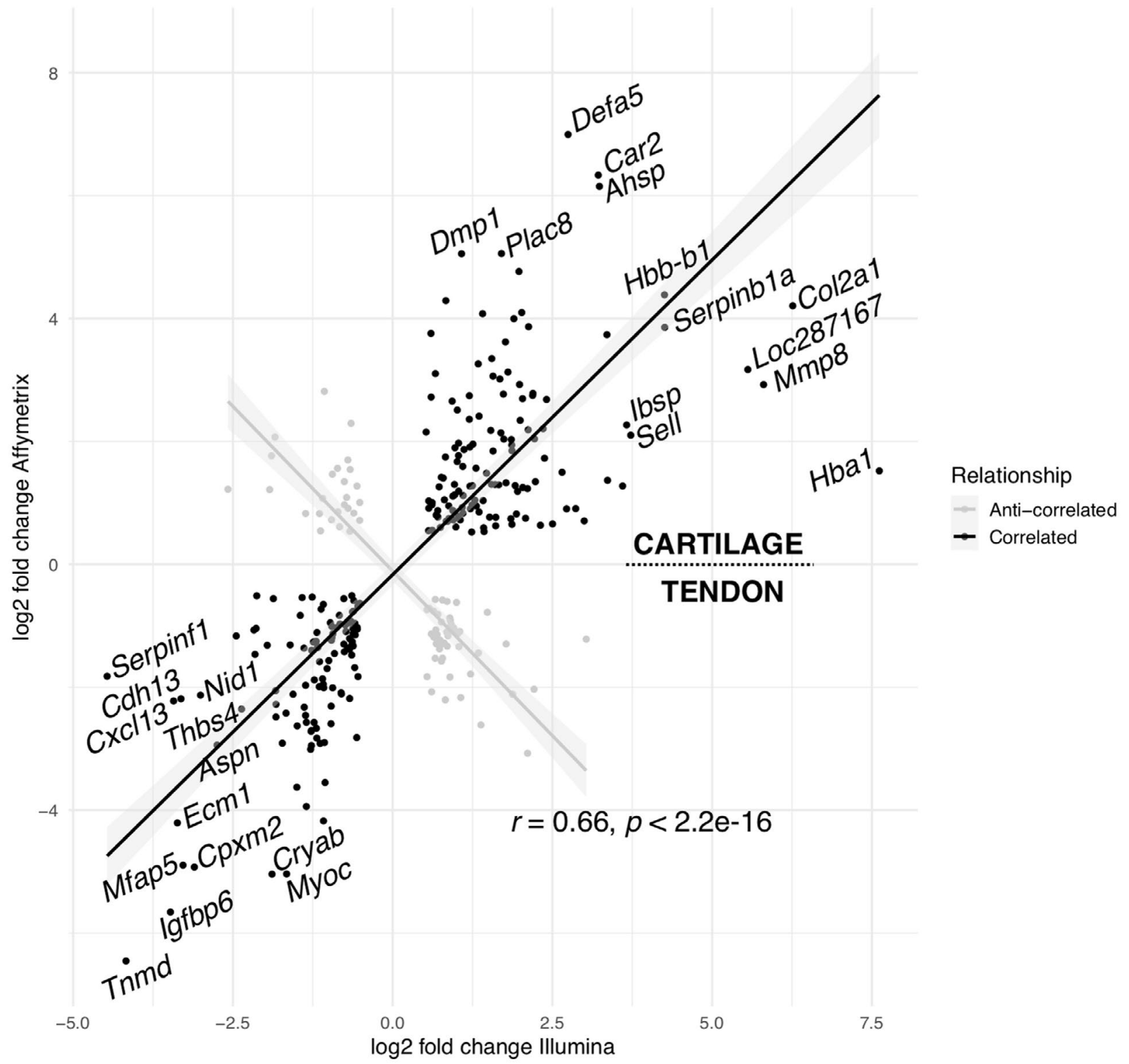

Fig. 1. Correlation plot of $\log _{2}$ fold changes of independent gene expression profiling studies of rat tendons. Genes found to be differentially expressed between native cartilage (top right quadrant) and tendons (bottom left quadrant) in both Illumina (x-axis) and Affymetrix (y-axis) datasets plotted by $\log _{2}$ fold-change. For clarity only, some data points are annotated. Data points are defined as correlated or anti-correlated in the relationship key. Where genes have the same directional change, the data point is defined as a dark dot, whereas genes with conflicting (anti-correlated) expression changes are defined as grey points (see relationship legend). For tendons, Tnmd, Igfbp6, Serpin1, Mfap5 and Ecm1 are all highly expressed in two independent datasets.

assessed for specificity by melting curve analysis. Gene expression levels were normalised to reference genes, using the efficiency corrected comparative $\mathrm{C}_{t}$ method (Pfaffl, 2006), with all qPCR reactions performed in triplicate. Reference genes ribosomal protein S20 (Rps20) and ribosomal protein L13a (Rpl13a) were shown to have the most stable expression across sample types in rat using the geNorm algorithm (Vandesompele et al., 2002) and geometric mean of their expression used for relative expression quantification in that species. Therefore, RPS20 was used as reference gene in sheep and horse. In human samples, glyceraldehyde 3-phosphate dehydrogenase (GAPDH) was identified as the most stable gene in all tissue types and used for relative expression quantification. Normalised $C_{t}$ data were converted to the linear form $\left(\mathrm{E}^{-\Delta \mathrm{Ct}}\right)$ for statistical analysis.

\section{Data analysis}

Following Shapiro-Wilks test for normality and Levene's tests for equality of variances, differences between conditions were analysed by one-way ANOVA with Tukey post-hoc test (equal variance between groups), Welch ANOVA with GamesHowell post-hoc test (unequal variance between groups) or Kruskal-Wallis test with Dunns pairwise comparison (non-parametric data). 
Results

Microarray analysis of rat tendon expression markers

Between rat cartilage and tendon gene expression profiles, 311 genes were commonly differentially expressed across two studies. Of these, 71 did not match in the direction of the fold change (decrease or increase in expression) between microarray platforms (Fig. 1). A moderately high correlation was observed between the fold changes of the 311 common genes (cor $=0.66, p<2.2 \mathrm{e}-16$ ). Genes that demonstrated the highest expression in tendon relative to cartilage in both studies included: Tmnd, Serpinf1, Igfbp6, Cpxm2, Cryab, Ecm1, Mfap5, Myoc, Aspn, Thbs4 (log fold change $>1.5$ ) (Table 2). Other tendon-related genes demonstrated more variable expression. A list of the top tendon-related genes in rat with their respective $\log _{2}$ fold-change values is presented in Table 3. When genes showing higher expression in tendon were functionally annotated using gene ontology terms, the following biological process and cell compartment terms were significant after adjustment for multiple testing $(p<0.05)$ : blood vessel development, developmental process, extracellular matrix. Interestingly, annotations specifically referencing tendon were not identified.

qPCR validation of putative markers

Validation of relevant tendon markers from a group of genes defined in the correlation study was undertaken relative to both cartilage and skeletal muscle in rats. This represented a third independent cohort. Genes selected for validation were inferred from correlation plot (Fig. 1) and highlighted in Table 3. Expression of developmental tendon markers $S c x$ and $M u s t n 1$ was additionally investigated alongside target genes derived from the microarray experiment. Significantly higher $(p<0.05)$ expression in tendon as compared to cartilage and muscle was detected for Aspn, Cpxm2, Ecm1, Igfbp6, Mfap5, Myoc, Serpinf1, Tnmd and Thbs4. Expression of the tendon development gene SCX was increased relative to cartilage, but not to muscle. Mustn1 and Cryab expression was similar in tendon as compared to other musculoskeletal tissues.

Table 2. Primer sequences for custom-designed oligonucleotides.

\begin{tabular}{|c|c|c|}
\hline Gene & Forward & Reverse \\
\hline \multicolumn{3}{|c|}{ Rat } \\
\hline Aspn & CCGAAAGGACTACCACCAACT & TTGTTTCCAAGACCCAGCCT \\
\hline Cpxm2 & GGCCTATGAAGGAGGTTCCG & CTCCCAGAGCAGCGTGTTTA \\
\hline Cryab & AAGAGCGCCAGGACGAAC & ACTCCATCCGATGACAGGGA \\
\hline Ecm1 & CCGTGACCAGTTCTTACCCC & CTGAAACCTTGAAGGCTCCCT \\
\hline Igfbp6 & CCGTCGGAAGAGACTACCAAG & CTTGAACAGGACTGGGCCTT \\
\hline Mfap5 & GCCAACGAGGAGATGACGTA & GTCATCCGTGGAAGGTGTGA \\
\hline Mustn1 & TTGCCTGTGGCTACTGCCTGC & GAGTGCCAGCCTCGGACATGG \\
\hline Myoc & GAGGGAGACAAAGGATGTGGAG & CAGTGATTGTCTCAGCTGTCCT \\
\hline$S c x$ & CCAGAGACGGCGGCGAGAAC & TTGGCTGCTGTGGACCCTCCT \\
\hline Serpinf1 & TGCCTTACTTCAAGGGGCAG & GTCCTGTCCTCGTCCAGGTG \\
\hline Thbs4 & AGTACCGCTGTAACGACACC & GGTTTGGGCGTTTGAGAAGG \\
\hline Tnmd & CCGCCGCACCAGACAAGCAA & GGCAGTAGCGGTTGCCTCGAC \\
\hline Rps20 & CCGCTGTTCGCTCCTGCTGA & TGCGGCTGGTGAGCGTGATT \\
\hline \multicolumn{3}{|c|}{ Sheep } \\
\hline$A S P N$ & GATCTGCAAAGGCTGGGTCT & TCACACGTGGTATGTTAGCAAGA \\
\hline ECM1 & GCTGTGCTGAGGAGGAGAAAT & GCAGAGTCTCGCCAGAAGTT \\
\hline IGFBP6 & AGGAACTCGGGGACCTCTAC & CAGCACGGAGTCCAGATGTT \\
\hline THBS4 & GACACCAGAGACGGCTTTCA & GGGGTGGTACTTGCACTCAT \\
\hline TNMD & ATCCСACTCTAATAGCAGTTTCAGA & CACCCACTGCTCGTTTTGTT \\
\hline RPS20 & GAAGGTGTGTGCTGACCTGA & CTCAGAGTCTTGGTAGGCATCC \\
\hline \multicolumn{3}{|c|}{$\begin{array}{rr}\text { Horse } \\
\end{array}$} \\
\hline$A S P N$ & ACGCTTTACACGTTCTGGAGAT & CTCCTTCAAATGCCCCTGGT \\
\hline ECM1 & CCAGGGTGAGACCCTCAATTT & GCATCCTCCCACACGAGTT \\
\hline IGFBP6 & GAACCGCAGAGACCAACAGA & ACGGGCCCATCTCCGT \\
\hline THBS4 & AATCCTGACAGACCCCACCC & GGTAGCGGAGGATGGCTTTGTT \\
\hline RPS20 & TTTGGAGAAGGTGTGTGCTGA & GTCTTGGTGGGCATCCGAA \\
\hline \multicolumn{3}{|c|}{$\begin{array}{ll}\text { Human } \\
\end{array}$} \\
\hline THBS4 & GTTGCAGAACCTGGCATTCAG & CCCTGGACCTGTCTTAGACTTCA \\
\hline GAPDH & ATGGGGAAGGTGAAGGTCG & TAAAAGCAGCCCTGGTGATGACC \\
\hline
\end{tabular}


Table 3. Top 40 differentially expressed genes in rat tendon as compared to cartilage resulting from two independent microarray studies. Only the genes with matching direction of $\log _{2}$ fold change between platforms were included. Genes highlighted in bold were selected for validation with RT-qPCR in rat tendon, cartilage and muscle.

\begin{tabular}{|c|c|c|c|}
\hline \multirow{2}{*}{$\begin{array}{l}\text { Gene } \\
\text { symbol }\end{array}$} & \multirow[b]{2}{*}{ Gene name } & \multicolumn{2}{|c|}{$\log _{2}$ fold change } \\
\hline & & Ilumina & Affymetrix \\
\hline Serpinf1 & Serpin family F member 1 & 4.4682858 & 1.8204873 \\
\hline Tnmd & Tenomodulin & 4.1729279 & 6.4517042 \\
\hline Igfbp6 & Insulin-like growth factor binding protein 6 & 3.4772336 & 5.6566756 \\
\hline Cxcl13 & Chemokine (C-X-C motif) ligand 13 & 3.4282285 & 2.222346 \\
\hline$E c m 1$ & Extracellular matrix protein 1 & 3.3686932 & 4.2056954 \\
\hline Cdh13 & Cadherin 13 & 3.3125358 & 2.1872266 \\
\hline Mfap5 & Microfibril associated protein 5 & 3.2807773 & 4.8933617 \\
\hline Cpxm2 & Carboxypeptidase X, M14 family member 2 & 3.103086 & 4.9246271 \\
\hline Nid1 & Nidogen 1 & 3.0072092 & 2.128184 \\
\hline Aspn & Asporin & 2.7472203 & 2.9400469 \\
\hline Lamc1 & Laminin subunit gamma 1 & 2.4477262 & 1.164511 \\
\hline Thbs4 & Thrombospondin 4 & 2.36365 & 2.3545135 \\
\hline Clic5 & Chloride intracellular channel protein 5 & 2.1707479 & 1.0681173 \\
\hline Lama4 & Laminin subunit alpha 4 & 2.1538213 & 1.463447 \\
\hline Has1 & Hyaluronan synthase 1 & 2.1369792 & 1.0421126 \\
\hline Nexn & Nexilin & 2.1266246 & 0.5113326 \\
\hline Cd151 & Cluster of differentiation 151 & 1.9624259 & 1.316076 \\
\hline Cryab & Crystallin alpha b & 1.8879595 & 5.0390885 \\
\hline Meox2 & Mesenchyme homeobox 2 & 1.8666559 & 0.5582083 \\
\hline Ccdc3 & Coiled-coil domain containing 3 & 1.8304224 & 2.0541286 \\
\hline Prss23 & Serine protease 23 & 1.8265794 & 2.4828796 \\
\hline S100a4 & S100 calcium binding protein $\mathrm{A} 4$ & 1.8254947 & 2.2765567 \\
\hline Itgbl1 & Integrin subunit beta like 1 & 1.7264565 & 2.909726 \\
\hline Crip2 & Cysteine rich protein 2 & 1.6683555 & 2.4204807 \\
\hline Myoc & Myocilin & 1.6593264 & 5.0361836 \\
\hline Agtr1a & Angiotensin II receptor type 1a & 1.6040215 & 1.3105763 \\
\hline Lmcd1 & Lim and cysteine rich domains 1 & 1.5583528 & 2.1135088 \\
\hline $\mathrm{Nbl1}$ & NBL1, DAN family BMP antagonist & 1.4990361 & 3.6254119 \\
\hline Fbln 2 & Fibulin-2 & 1.4933224 & 2.6278954 \\
\hline Rgs4 & Regulator of $\mathrm{G}$ protein signaling 4 & 1.4458768 & 0.8312598 \\
\hline Cygb & Cytoglobin & 1.4163564 & 0.5405094 \\
\hline Sncg & Synuclein gamma & 1.3867141 & 1.3627389 \\
\hline Procr & Protein C receptor & 1.3833375 & 2.3241227 \\
\hline Thbd & Thrombomodulin & 1.3626751 & 2.4577927 \\
\hline Chrnb1 & Cholinergic receptor nicotinic beta 1 subunit & 1.3621048 & 1.9674221 \\
\hline Ltbp4 & Latent transforming growth factor $\beta$ binding protein 4 & 1.3502595 & 3.938885 \\
\hline Lamb2 & Laminin subunit beta 2 & 1.3499291 & 2.5700232 \\
\hline$C f b$ & Complement factor $\mathrm{b}$ & 1.2835458 & 3.008987 \\
\hline Lama2 & Laminin subunit alpha 2 & 1.2763259 & 2.717257 \\
\hline Mustn1 & Musculoskeletal, embryonic nuclear protein 1 & 1.2715101 & 2.9484326 \\
\hline
\end{tabular}

Tendon marker analysis in human and model species

Genes showing the highest expression in tendon (ASPN, ECM1, IGFBP6, TNMC and THBS4), with $p<0.001$, were selected for further evaluation in sheep and horse (Fig. 2a). Unlike in rat, ECM1 expression was higher in cartilage for both sheep and horse; $A S P N$ had high expression in horse tendon, but not in sheep. Therefore, only IGFBP6,
TNMD and THBS4 were additionally analysed in human musculoskeletal tissues (Fig. 2a). Expression of THBS4 was highly variable but showed a trend towards higher expression in all species' tendons. Higher expression of TNMD was confirmed in rat, sheep and human but not equine tendon. Only IGFBP 6 demonstrated significantly higher expression in tendon relative to cartilage and muscle in all species (Fig. 2a). Therefore, nucleotide sequence 
identity of IGFBP 6 across the species was evaluated using NCBI BLAST (Altschul et al., 1997) (Fig. 2b).

IGFBP6 in equine tendon with relation to function and age

Expression of IGFBP6 in a region of equine SDFT subjected to compressive force and, thus, displaying more fibrocartilage-like morphology was lower than in the SDFT tensional midportion (Fig. 3b). No significant difference in expression was observed between energy-storing (SDFT) and positional tendon (CDET), with SDFT expression results showing bigger individual variation than CDET (Fig. 3c). IGFBP6 expression increased between $0 \mathrm{~d}$ and 12-24 months of age, followed by stabilisation at lower level in the older age groups (3-5, 8-11 and 18-22 years); however, none of those age-related differences achieved statistical significance (Fig. 3d).

\section{Discussion}

Evaluation of bioengineered tendon constructs and native tendon tissue is based predominantly on histological examination and a limited pool of molecular markers often not tested for potential species-, age- and function-related differences among tendons. In the present study, a group of genes, identified by unbiased microarray analysis in rat native tendon and primary tenocytes, was validated across several species relevant for tendon studies and confirmed in human. Among those targets were genes previously recognised as tendon-specific (TNMD, THBS4) (Docheva et al., 2005; Hauser et al., 1995; Jelinsky et al., 2010) or known to be expressed in tendon but not subjected to direct comparisons with other adult musculoskeletal tissues (IGFBP6, ASPN, ECM1) (Dahlgren and Nixon, 2005; Henry et al., 2001; Jelinsky et al., 2010; Kuntz et al., 2018). It is worth noting that potential markers identified were limited to genes included in the microarray platforms and other tendon-specific genes could be identified should a similar approach be employed using RNA sequencing.

Primarily, candidate tendon markers should be able to differentiate clearly between tendon and cartilage as chondrogenic transformation is a common feature of degenerated tendon (Asai et al., 2014; Burssens et al., 2013; Clegg et al., 2007; Titan and Andarawis-Puri, 2016). ECM1 and ASPN, although highly expressed in rat tendons, showed similar or higher expression level in sheep and horse cartilage, thus reducing their usability as tendon markers. That ambiguity in gene expression is in concordance with previous studies. ECM1 is more expressed in porcine Achilles tendon enthesis (tendon-bone
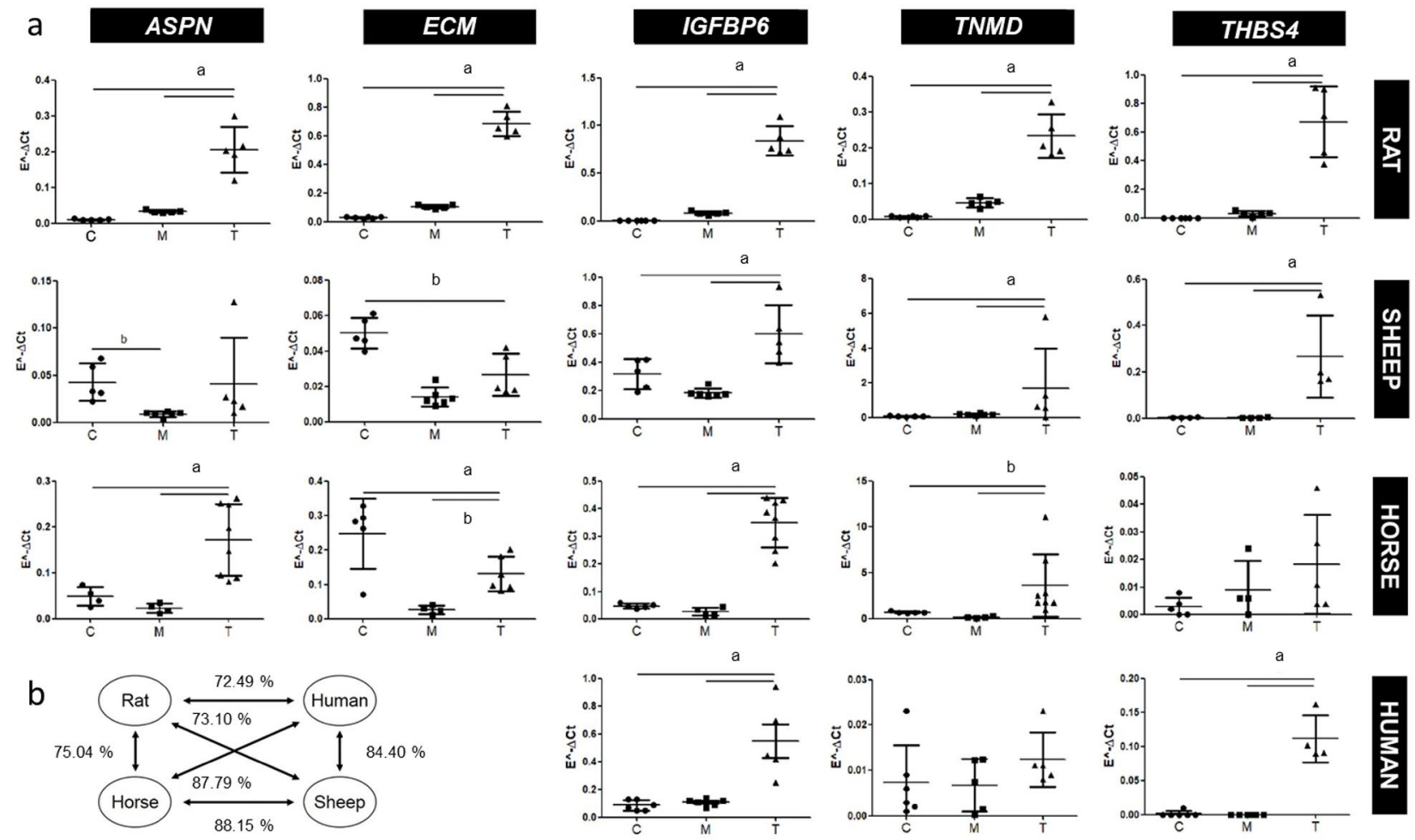

Fig. 2. RT-qPCR validation of candidate marker genes identified using microarray analysis across musculoskeletal tissues in rat, sheep, horse and human. (a) Data were normalised to Rps20 and Rpl13 (rat), RPS20 (horse and sheep) and GAPDH (human) and presented as efficiency corrected $2^{-\Delta C t}$. Bars represent the average expression and standard deviation for each group. A significant difference between average expression in cartilage (C), muscles (M) and tendons (T) was marked as: ${ }^{\mathrm{a}} p<0.001,{ }^{\mathrm{b}} p<0.05$ as determined using one-way (equal variances) or Welch (unequal variances) ANOVA. (b) Nucleotide sequence identity of IGFBP6 across the species investigated determined as statistically significant by NCBI BLAST (E-value $<1 \mathrm{e}-92$ ). 
insertion) than tendon body (Kuntz et al., 2018) and $A S P N$ in human adult cartilage, although with no reference to expression levels in other tissues (Henry et al., 2001). Interestingly, SCX used in the rat RTqPCR experiment as a benchmark tendon marker (not derived from the current microarray analysis) showed substantial variability between individual tendon samples, not observed in muscle or cartilage and contributing to loss of significance when comparing average expression among tissues (Fig. 2a). Similarly, Mohawk (MKX) gene, known to be critical in tendon development, despite being present in both microarray platforms, was not significantly higher in tendons than in cartilage. SCX is known for its key role in tendon development and differentiation (Schweitzer et al., 2001); however, its expression in tendons declines with their maturation (Chen et al., 2017) and can be affected by tendon mechanical environment (Murchison et al., 2007; Scott et al., 2011). Therefore, it is possible that SCX expression in adult tendons is more susceptible to differences in local biomechanical factors or individual activity levels. $S C X$ regulates expression of another tendon marker investigated, TNMD (Shukunami et al., 2006). Results confirmed tendon-specific expression of TNMD in all species apart from horse, where high variability among biological replicates and no clear difference in expression among tissues were found (Fig. 2a). This finding is supported by previous equine tendon marker studies (Kuemmerle et al., 2016; Taylor et al., 2009) and may challenge the frequent application of TNMD as a marker of tendon healing and tenogenic differentiation of stem cells in horse (Barsby and Guest, 2013; Durgam et al., 2016; Smith et al., 2015). Regulation of TNMD transcription is complex and involves SCX-independent mechanisms, such as Wnt/ $\beta$-catenin signalling (Katsuhiko et al., 2014), which is responsive to mechanical stimulation in other musculoskeletal tissues (Lara-Castillo et al., 2015; Niu et al., 2016), potentially enhancing individual differences in TNMD mRNA levels. The reason for variable expression of THBS4 in human musculoskeletal tissues in the present study was not clear. THBS4 may be abundantly expressed in muscle (Frolova et al., 2014); however, it is expected to show good separation between tendon and cartilage (Jelinsky et al., 2010). Recent work [Simpson et al., (2019) Isotopic labelling reveals diverse rate of protein turnover within tendon. In: 2019 Annu Meet Orthop Res Soc Austin, TX, USA] on use of isotopic labelling for evaluation of protein turnover rate within tendons has demonstrated that THBS4 is the most quickly metabolised ECM protein, which perhaps is also reflected by fluctuations at the gene

\section{IGFBP6}

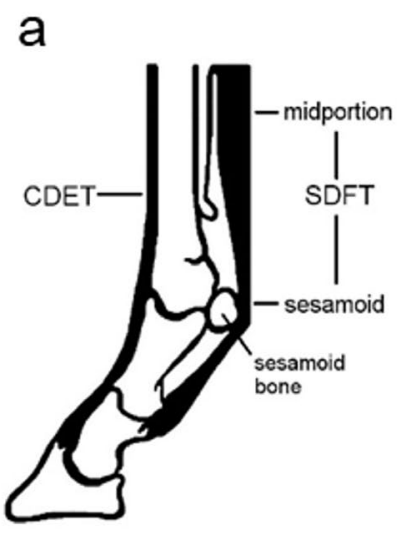

b

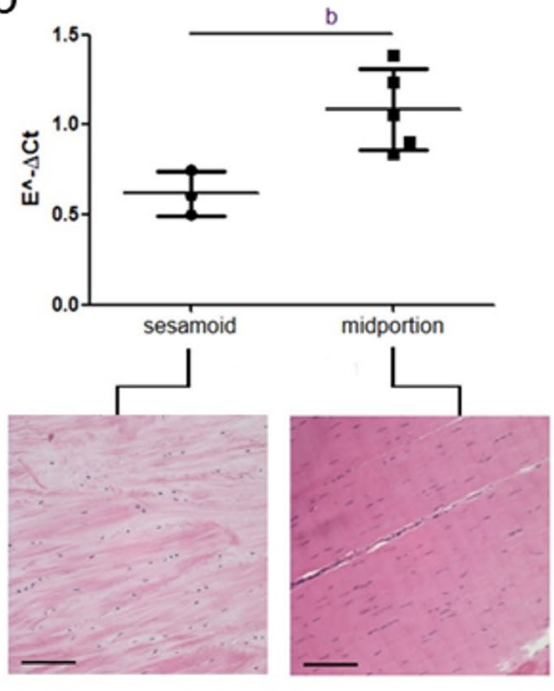

C

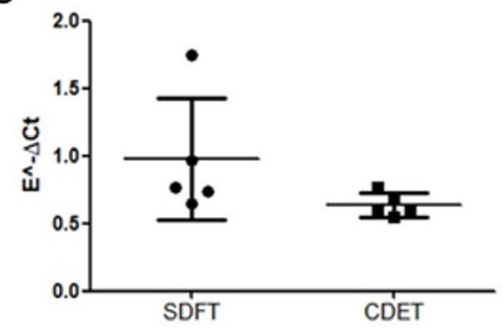

d

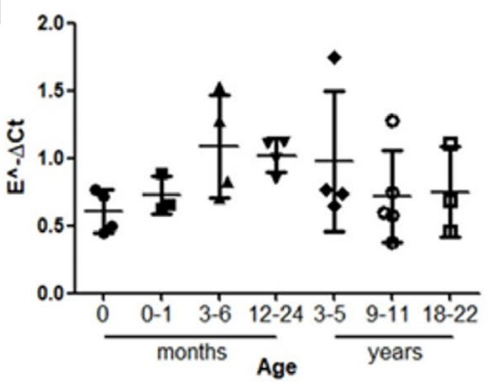

Fig. 3. The effect of age and anatomical location on IGFBP6 expression in equine tendons determined by RT-qPCR. (a) Schematic view of investigated structures in equine distal limb (tendons marked in black). CDET function is to stabilise the limb joints (positional tendon) while SDFT plays a role in locomotion (energy-storing tendon). SDFT sesamoid region is subjected to compression by adjacent proximal sesamoid bones affecting tendon morphology. Expression of IGFBP6 was compared in (b) two regions of SFDT sesamoid and midportion. Images below the graph present differences in histological structure of the two regions (haematoxylin and eosin stain, scale bar $=100 \mu \mathrm{m}$ ); $(\mathbf{c})$ functionally distinct tendons, SDFT and CDET; (d) SDFT during postnatal development and ageing. Data were normalised to RPS20 and presented as efficiency corrected $2^{-\triangle C t}$. Bars represent the average expression and standard deviation for each group. Significant difference between average expression in each group was marked as: ${ }^{\mathrm{a}} p<0.001,{ }^{\mathrm{b}} p<0.05$ as determined using $(\mathbf{a}, \mathbf{b}) t$-test or $(\mathbf{c})$ Kruskal-Wallis test with Dunns pairwise comparisons. 
expression level. Results discussed above add to the general conclusion that species can have a significant effect on tendon gene expression and that an optimal tendon marker should facilitate translation between animal experiments and human clinical studies.

Among the initial pool of candidate tendon markers, IGFBP6 was the only gene consistently more expressed in tendons as compared to cartilages and muscles across different species. This finding, combined with high nucleotide-sequence identity (Fig. 2b), may suggest IGFBP6 homology and conservation of its evolutionary function across species. Expression of IGFBP 6 in tendon has previously been shown in a human microarray study comparing a wider group of tissue types including bone, bone marrow, cartilage, fat and muscle (Jelinsky et al., 2010). IGFBP6 is the main regulator of insulin growth factor II (IGF-II) availability and it also displays several IGF-II-independent actions affecting cell proliferation, survival, differentiation and migration (Bach, 2016). IGFs stimulate proteoglycan, collagen and non-collagen synthesis in flexortendon-derived cells (Abrahamsson, 1997) and causes cell proliferation in both the epitenon and endotenon (Murphy and Nixon, 1997). Thus, proteins regulating their activity may have a significant role in ECM remodelling relevant for tendon physiology. Decreased expression of IGFBP 6 was described in an equine model of tendon injury and human fibroblasts from Dupuytren's disease tissue (Dahlgren et al., 2006; Raykha et al., 2013) and altered methylation of IGFBP 6 promoter was detected in a chemical model of murine tendinopathy (Trella et al., 2017). In the present study, fibrocartilaginous transformation of the equine SDFT region compressed by sesamoid bones (similar to patellar compression of quadriceps femoris tendon in humans) was associated with lower IGFBP6 expression (Fig. 3), indicating that not only can it discriminate between different mesenchymal tissue lineages but also react to phenotype change within fully differentiated tendon tissue. A trend for increasing levels of IGFBP6 in SDFT postnatal development could reflect gradual restriction of growth factor accessibility corresponding with reaching musculoskeletal maturity (two years of age in the horse), when IGFBP6 level stabilised (Fig. 3d). The lack of a significant effect of ageing or function (positional versus energy-storing tendon) on IGFBP6 expression suggests its robustness as a biomarker of tendon phenotype, which may be desirable in the context of a practical application. In the light of the current evidence, IGFBP 6 expression appears to be useful in recognition of a functional tendon phenotype and may potentially become employed in evaluating tendon degeneration in intra-operative and biopsy tendon samples. However, that would require further investigation of IGFBP6 expression in fibrous scar tissue resulting from tendon injuries, for instance by analysing overload lesions frequently diagnosed in human and equine athletes. Fibrous tissue produced during tendon healing has altered microstructure and ECM composition (e.g. increased proportion of type III to type I collagen) which results in compromised mechanical properties and high risk of re-injury (Yang et al., 2014). A gene marker differentiating intact from repair tissue could inform decision on the extent of tendon dissection during surgical management of shoulder tendon injuries. Another important direction in tendon marker application is quality assessment of engineered tissues for use in tendon lesion repair. Previously published datasets comparing transcriptomic profiles of rat tenocytes and chondrocytes maintained in different culture systems and in native tissue (Mueller et al., 2016) showed that IGFBP6 expression in tendon-derived cells is consistently higher than in those derived from cartilage, independently of the culture method [monolayer or three-dimensional (3D) tissue construct]. Particularly interesting is that IGFBP6 maintained higher expression in late passage monolayer tenocytes despite the general loss of a tissue-specific transcriptomic signature. Dedicated studies investigating IGFBP6 at different stages of tendon construct creation and relating the expression level to structural and functional outcomes are needed to validate its use in directed tendon construct differentiation.

Limitations associated with the study, mainly pertained to collection of a sample range from different tissue types and species. Unlike rat, sheep and horse, human tendon, cartilage and muscle samples were collected from different donor groups, with muscle donors being considerably younger than cartilage and tendon donors. That difference is related to the fact that healthy cartilage and tendons for research are mainly derived from cadaveric sources, while muscles can be collected intraoperatively or as biopsy samples. Age disparity could have potentially affected comparability of expression results from different musculoskeletal tissues. Nevertheless, two studies analysing age-related changes in human tendon and cartilage transcriptome by RNA sequencing did not identify any of the marker genes investigated in the present study to be differentially expressed between young old subjects (Peffers et al., 2013; 2015). Results of IGFBP6 analysis in horse further demonstrated that growth and development are more likely to affect expression of tendon marker genes than ageing. Another potential source of variation in gene expression levels was the circadian rhythm, as it was not possible to control for sample collection time in most species, except for rats. The available studies investigating circadian changes in mouse tendon indicated that only THBS4 expression is rhythmic (Yeung et al., 2014). It is also unclear if species considered in the present study have the same tendon circadian clock. Additionally, analysis of bone alongside tendon, cartilage and muscle would be desirable as ectopic ossification is one of the manifestations of tendon degeneration (Agarwal 
et al., 2017). However, it is suspected that appearance of osteoblasts, responsible for tissue mineralisation in tendon, is preceded by fibrocartilaginous metaplasia of tenocytes. According to the present study, that change is associated with a marked decrease in IGFBP6 expression level. IGFBP6 has been also shown to be involved in mechanisms inhibiting human osteoblast phenotype development (Yan et al., 2001). Whilst IGFBP6 expression appears to be a reliable marker of tendon tissue, similar relations may not necessarily occur at the protein level. Validation of the results described in the present study would be required to introduce the use of IGFBP 6 in proteinbased assays, opening additional avenues for its clinical application. In order to establish IGFBP6 as clinical marker of healthy tendon phenotype, present results would need to be validated by an independent cohort study comparing the effect of age, sex and pathologies on IGFBP6 expression.

\section{Conclusion}

IGFBP6 was identified as a universal transcriptomic marker in rat, sheep, equine and human tendons and may constitute a potential reference biomarker for evaluation of tendon physiological phenotype and directed development of engineered tendons.

\section{Acknowledgements}

We would like to acknowledge Dr Mandy Peffers, Dr Kasia Goljanek-Whysall and Dr Yalda Ashraf-Kharaz for their help with obtaining samples and reagents. We would like to thank the staff of the Department of Veterinary Pathology and Public Health, Institute of Veterinary Science at the University of Liverpool for assistance with sample collection. The study was funded by BBSRC Doctoral Training Grant, Erasmus+ and Wellcome Trust Institution Strategic Support Fund to the University of Liverpool.

\section{References}

Abrahamsson SO (1997) Similar effects of recombinant human insulin-like growth factor-I and II on cellular activities in flexor tendons of young rabbits: experimental studies in vitro. J Orthop Res 15: 256-262.

Agarwal S, Loder SJ, Cholok D, Peterson J, Li J, Breuler C, Cameron Brownley R, Hsin Sung H, Chung MT, Kamiya N, Li S, Zhao B, Kaartinen V, Davis TA, Qureshi AT, Schipani E, Mishina Y, Levi B (2017) Scleraxis-lineage cells contribute to ectopic bone formation in muscle and tendon. Stem Cells 35: 705-710.

Altschul SF, Madden TL, Schaffer AA, Zhang J, Zhang Z, Miller W, Lipman DJ (1997) Gapped BLAST and PSI-BLAST: a new generation of protein database search programs. Nucleic Acids Res 25: 3389-3402.

Andarawis-Puri N, Flatow EL (2011) Tendon fatigue in response to mechanical loading. J Musculoskelet Neuronal Interact 11: 106-114.

Asai S, Otsuru S, Candela ME, Cantley L, Uchibe K, Hofmann TJ, Zhang K, Wapner KL, Soslowsky LJ, Horwitz EM, Enomoto-Iwamoto M (2014) Tendon progenitor cells in injured tendons have strong chondrogenic potential: the CD105-negative subpopulation induces chondrogenic degeneration. Stem Cells 32: 3266-3277.

Bach LA (2016) Current ideas on the biology of IGFBP-6: more than an IGF-II inhibitor? Growth Horm IGF Res 30-31: 81-86.

Barsby T, Guest D (2013) Transforming growth factor beta3 promotes tendon differentiation of equine embryo-derived stem cells. Tissue Eng Part A 19: 2156-2165.

Brunton B, Suksanpaisan L, Li H, Liu Q, Yu Y, Vrieze A, Zhang L, Jenks N, Jiang H, DeGrado TR, Zhao C, Russell SJ, Peng KW (2018) New transgenic NIS reporter rats for longitudinal tracking of fibrogenesis by high-resolution imaging. Sci Rep 8: 14209. DOI: $10.1038 / s 41598-018-32442-x$.

Burssens A, Forsyth R, Bongaerts W, Jagodzinski M, Mahieu N, Praet M, Victor J (2013) Arguments for an increasing differentiation towards fibrocartilaginous components in midportion Achilles tendinopathy. Knee Surg Sports Traumatol Arthrosc 21: 1459-1467.

Chen J, Zhang E, Zhang W, Liu Z, Lu P, Zhu T, Yin Z, Backman LJ, Liu H, Chen X, Ouyang H (2017) Fos promotes early stage teno-lineage differentiation of tendon stem/progenitor cells in tendon stem cells. Transl Med 6: 2009-2019.

Chomczynski P, Sacchi N (2006) The single-step method of RNA isolation by acid guanidinium thiocyanate-phenol-chloroform extraction: twentysomething years on. Nat Protoc 1: 581-585.

Clegg PD, Strassburg S, Smith RK (2007) Cell phenotypic variation in normal and damaged tendons. Int J Exp Pathol 88: 227-235.

Dahlgren LA, Van Der Meulen MCH, Bertram JEA, Starrak GS, Nixon AJ (2002) Insulin-like growth factor-I improves cellular and molecular aspects of healing in a collagenase-induced model of flexor tendinitis. J Orthop Res 20: 910-919.

Dahlgren LA, Mohammed HO, Nixon AJ (2006) Expression of insulin-like growth factor binding proteins in healing tendon lesions. J Orthop Res 24: 183-192.

Dahlgren LA, Nixon AJ (2005) Cloning and expression of equine insulin-like growth factor binding proteins in normal equine tendon. Am J Vet Res 66: 300-306.

Docheva D, Hunziker EB, Fassler R, Brandau O (2005) Tenomodulin is necessary for tenocyte proliferation and tendon maturation. Mol Cell Biol 25: 699-705.

Durgam SS, Stewart AA, Sivaguru M, Wagoner Johnson AJ, Stewart MC (2016) Tendon-derived 
progenitor cells improve healing of collagenaseinduced flexor tendinitis. J Orthop Res 34: 2162-2171.

Frolova EG, Drazba J, Krukovets I, Kostenko V, Blech L, Harry C, Vasanji A, Drumm C, Sul P, Jenniskens GJ, Plow EF, Stenina-Adognravi O (2014) Control of organization and function of muscle and tendon by thrombospondin-4. Matrix Biol 37: 35-48.

Harris MT, Butler DL, Boivin GP, Florer JB, Schantz EJ, Wenstrup RJ (2004) Mesenchymal stem cells used for rabbit tendon repair can form ectopic bone and express alkaline phosphatase activity in constructs. J Orthop Res 22: 998-1003.

Hauser N, Paulsson M, Kale AA, DiCesare PE (1995) Tendon extracellular matrix contains pentameric thrombospondin-4 (TSP-4). FEBS Lett 368: 307-310.

Hausmann JT, Vekszler G, Bijak M, Benesch T, Vécsei V, Gäbler C (2009) Biomechanical comparison of modified Kessler and running suture repair in 3 different animal tendons and in human flexor tendons. J Hand Surg Am 34: 93-101.

Henry SP, Takanosu M, Boyd TC, Mayne PM, Eberspaecher H, Zhou W, De Crombrugghe B, Höök M, Mayne R (2001) Expression pattern and gene characterization of asporin. A newly discovered member of the leucine-rich repeat protein family. J Biol Chem 276: 12212-12221.

Howell K, Chien C, Bell R, Laudier D, Tufa SF, Keene DR, Andarawis-Puri N, Huang AH (2017) Novel model of tendon regeneration reveals distinct cell mechanisms underlying regenerative and fibrotic tendon healing. Sci Rep 7: 45238. DOI: 10.1038/ srep45238.

Jelinsky SA, Archambault J, Li L, Seeherman H (2010) Tendon-selective genes identified from rat and human musculoskeletal tissues. J Orthop Res 28: 289-297.

Katsuhiko A, Atsutoshi K, Yohei Y, Yoshinori K, Shihori M (2014) Regulation of tenomodulin expression via $\mathrm{wnt} / \beta$-catenin signaling in equine bone marrow-derived mesenchymal stem cells. J Equine Sci 25: 7-13.

Kjær M, Langberg $H$, Heinemeier $K$, Bayer ML, Hansen M, Holm L, Doessing S, Kongsgaard M, Krogsgaard MR, Magnusson SP (2009) From mechanical loading to collagen synthesis, structural changes and function in human tendon. Scand J Med Sci Sport 9: 500-510.

Kuemmerle JM, Theiss F, Okoniewski MJ, Weber FA, Hemmi S, Mirsaidi A, Richards PJ, Cinelli P (2016) Identification of novel equine (Equus caballus) tendon markers using RNA sequencing. Genes (Basel) 7: E97. DOI: 10.3390/genes7110097.

Kuntz LA, Rossetti L, Kunold E, Schmitt A, von Eisenhart-Rothe R, Bausch AR, Burgkart RH (2018) Biomarkers for tissue engineering of the tendonbone interface. PLoS One 13: e0189668. DOI: 10.1371/ journal.pone.0189668.

Lara-Castillo N, Kim-Weroha NA, Kamel MA, Javaheri B, Ellies DL, Krumlauf RE, Thiagarajan G, Johnson ML (2015) In vivo mechanical loading rapidly activates $\beta$-catenin signaling in osteocytes through a prostaglandin mediated mechanism. Bone 76: 58-66.

Lui PPY, Maffulli N, Rolf C, Smith RKW (2011) What are the validated animal models for tendinopathy? Scand J Med Sci Sport 21: 3-17.

Mehr D, Pardubsky PD, Martin JA, Buckwalter JA (2000) Tenascin-C in tendon regions subjected to compression. J Orthop Res 18: 537-545.

Mueller AJ, Tew SR, Vasieva O, Clegg PD, Canty-Laird EG (2016) A systems biology approach to defining regulatory mechanisms for cartilage and tendon cell phenotypes. Sci Rep 6: 33956. DOI: 10.1038/srep33956.

Müller SA, Todorov A, Heisterbach PE, Martin I, Majewski M (2013) Tendon healing: an overview of physiology, biology, and pathology of tendon healing and systematic review of state of the art in tendon bioengineering. Knee Surg Sports Traumatol Arthrosc 23: 2097-2105.

Murchison ND, Price BA, Conner DA, Keene DR, Olson EN, Tabin CJ, Schweitzer R (2007) Regulation of tendon differentiation by scleraxis distinguishes force-transmitting tendons from muscle-anchoring tendons. Development 134: 2697-2708.

Murphy DJ, Nixon AJ (1997) Biochemical and site-specific effects of insulin-like growth factor I on intrinsic tenocyte activity in equine flexor tendons. Am J Vet Res 58: 103-109.

Niu Q, Li F, Zhang L, Xu X, Liu Y, Gao J, Feng X (2016) Role of the Wnt/-catenin signaling pathway in the response of chondrocytes to mechanical loading. Int J Mol Med 37: 755-762.

Patterson-Kane JC, Becker DL, Rich T (2012) The pathogenesis of tendon microdamage in athletes: the horse as a natural model for basic cellular research. J Comp Pathol 147: 227-247.

Peffers M, Fang Y, Cheung K, Wei T, Clegg P, Birch H (2015) Transcriptome analysis of ageing in uninjured human Achilles tendon. Arthritis Res Ther 17: 33. DOI: 10.1186/s13075-015-0544-2.

Peffers MJ, Liu X, Clegg PD (2013) Transcriptomic signatures in cartilage ageing. Arthritis Res Ther 15: R98. DOI: 10.1186/ar4278.

Peffers MJ, Thorpe CT, Collins JA, Eong R, Wei TKJ, Screen HRC, Clegg PD (2014) Proteomic analysis reveals age-related changes in tendon matrix composition, with age- and injury-specific matrix fragmentation. J Biol Chem 289: 25867-25878.

Pfaffl M (2006) Relative quantification. In: Realtime PCR. Editor: T. Dorak. International University Line. pp: 63-82.

Raykha C, Crawford J, Gan BS, Fu P, Bach LA, O'Gorman DB (2013) IGF-II and IGFBP-6 regulate cellular contractility and proliferation in Dupuytren's disease. Biochim Biophys Acta 1832: 1511-1519.

Ritty TM, Roth R, Heuser JE (2003) Tendon cell array isolation reveals a previously unknown fibrillin-2-containing macromolecular assembly. Structure 11: 1179-1188.

Schweitzer R, Chyung JH, Murtaugh LC, Brent AE, Rosen V, Olson EN, Lassar A, Tabin CJ (2001) Analysis 
of the tendon cell fate using scleraxis, a specific marker for tendons and ligaments. Development 128: 3855-3866.

Scott A, Danielson P, Abraham T, Fong G, Sampaio AV, Underhill TM (2011) Mechanical force modulates scleraxis expression in bioartificial tendons. J Musculoskelet Neuronal Interact 11: 124-132.

Scott A, Backman LJ, Speed C (2015) Tendinopathy: update on pathophysiology. J Orthop Sport Phys Ther 45: 833-841.

Shukunami C, Takimoto A, Oro M, Hiraki Y (2006) Scleraxis positively regulates the expression of tenomodulin, a differentiation marker of tenocytes. Dev Biol 298: 234-247.

Smith LC, Smith O, Bavin EP, Guest DJ, Baird AEG (2015) Equine induced pluripotent stem cells have a reduced tendon differentiation capacity compared to embryonic stem cells. Front Vet Sci 2: 55. DOI: 10.3389/fvets.2015.00055.

Smyth GK (2005) Linear models and empirical bayes methods for assessing differential expression in microarray experiments stat. Appl Genet Mol Biol 3: Article3. DOI: 10.2202/1544-6115.1027.

Song L, Olsen RE, Spalazzi JP, Davisson T (2010) Biomechanical evaluation of acellular collagen matrix augmented Achilles tendon repair in sheep. J Foot Ankle Surg 49: 438-441.

Soriano-Arroquia A, Clegg PD, Molloy AP, Goljanek-Whysall K (2017) Preparation and culture of myogenic precursor cells/primary myoblasts from skeletal muscle of adult and aged humans. J Vis Exp 120. DOI:10.3791/55047.

Szaro P, Witkowski G, Ciszek B (2012) Anatomy of the common calcaneal tendon in rat (Rattus norvegicus). Polish J Nat Sci 27: 339-346.

Tan Q, Lui PPY, Rui YF (2012) Effect of in vitro passaging on the stem cell-related properties of tendon-derived stem cells - implications in tissue engineering. Stem Cells Dev 21: 790-800.

Taylor SE, Vaughan-Thomas A, Clements DN, Pinchbeck G, MacRory LC, Smith RK, Clegg PD (2009) Gene expression markers of tendon fibroblasts in normal and diseased tissue compared to monolayer and three dimensional culture systems. BMC Musculoskelet Disord 10: 27. DOI: 10.1186/14712474-10-27.

Testa S, Costantini M, Fornetti E, Bernardini S, Trombetta M, Seliktar D, Cannata S, Rainer A, Gargioli C (2017) Combination of biochemical and mechanical cues for tendon tissue engineering. J Cell Mol Med 21: 2711-2719.

Thorpe CT, Godinho MSC, Riley GP, Birch HL, Clegg PD, Screen HRC (2015) The interfascicular matrix enables fascicle sliding and recovery in tendon, and behaves more elastically in energy storing tendons. J Mech Behav Biomed Mater 52: 8594.

Thorpe CT, Screen HRC (2016) Tendon structure and composition. Adv Exp Med Biol 920: 3-10.

Titan A, Andarawis-Puri N (2016) Tendinopathy. JBJS Rev 4. DOI: 10.2106/JBJS.RVW.15.00088.
Trella KJ, Li J, Stylianou E, Wang VM, Frank JM, Galante J, Sandy JD, Plaas A, Wysocki R (2017) Genome-wide analysis identifies differential promoter methylation of Leprel2, Foxf1, Mmp25, Igfbp6, and Peg12 in murine tendinopathy. J Orthop Res 35: 947-955.

Vandesompele J, De Preter K, Pattyn F, Poppe B, Van Roy N, De Paepe A, Speleman F (2002) Accurate normalization of real-time quantitative RT-PCR data by geometric averaging of multiple internal control genes. Genome Biol 3: RESEARCH0034. DOI: 10.1186/ gb-2002-3-7-research0034.

Yan T, Wergedal J, Zhou Y, Mohan S, Baylink DJ, Strong DD (2001) Inhibition of human osteoblast marker gene expression by retinoids is mediated in part by insulin-like growth factor binding protein-6. Growth Horm IGF Res 11: 368-377.

Yang G, Rothrauf BB, Tuan RS (2014) Tendon and ligament regeneration and repair: clinical relevance and developmental paradigm. Birth Defects Res C Embryo Today 99: 203-222.

Yao L, Bestwick CS, Bestwick LA, Maffulli N, Aspden RM (2006) Phenotypic drift in human tenocyte culture. Tissue Eng 12: 1843-1849.

Ye J, Coulouris G, Zaretskaya I, Cutcutache I, Rozen S, Madden TL (2012) Primer-BLAST: a tool to design target-specific primers for polymerase chain reaction. BMC Bioinformatics 13: 134. DOI: 10.1186/1471-2105-13-134.

Yeung CYC, Gossan N, Lu Y, Hughes A, Hensman JJ, Bayer ML, Kjær M, Kadler KE, Meng QJ (2014) Gremlin-2 is a BMP antagonist that is regulated by the circadian clock. Sci Rep 4: 5183. DOI: 10.1038/ srep05183.

Zhang J, Pan T, Liu Y, Wang JHC (2010) Mouse treadmill running enhances tendons by expanding the pool of tendon stem cells (TSCs) and TSC-related cellular production of collagen. J Orthop Res 28: 11781183.

\section{Web Reference}

\section{1. https://www.r-project.org/ [16-09-19]}

\section{Discussion with Reviewers}

Linda Dahlgren: Do you think that IGFBP 6 would be differentially expressed between tendon and a general fibrous connective tissue? Or between tendon fibroblasts passaged in culture and primary tendon fibroblasts?

Authors: Gene expression profiles of rat primary tenocytes, tenocytes cultured in monolayer (passage 3) and 3D fibrin constructs (unpublished data, presented at British Society for Matrix Biology Spring Meeting, 2019) were compared. IGFBP6 was significantly more expressed $\left[\log _{2}\right.$ fold change $> \pm 0.5$, false disovery rate $(\mathrm{FDR})<0.01$, log-odds ratio of expression $>0$ ] in primary cells and monolayer 
culture than in 3D constructs. Also, higher expression of IGFBP 6 was observed in equine tendon cultured in monolayer (passage 3) than in 3D fibrin construct, as measured by RT-qPCR. Tenocytes cultured in monolayer lose their specific phenotype (Muller et al., 2016; Yao et al., 2006). Therefore, higher expression of IGFBP6 in primary than in cultured tenocytes could further support its importance as a tendon marker. However, it is unclear why monolayer cells would show higher IGFBP6 transcript level than tenocytes cultured in a 3D system showing more similarity to the native tissue in global expression profile (Yao et al., 2006). This observation might question IGFBP6 usability for tendon engineering purposes or the value of the applied 3D culture technique for tendon phenotype maintenance. It is likely that IGFBP6 declines with culture time as generating 3D bioengineered tendon requires significantly more time than reaching confluence in cell monolayer. Evaluating IGFBP6 expression in a range of tendon in vitro models, including novel multifactorial culture systems, is needed to validate its application as tendon marker for bioengineering application.

Brianne Connizzo: Is IGFBP6 expressed highly in other non-musculoskeletal tissues that might be present in vivo, such as vascular or adipose tissues? Authors: IGFBP6 is expressed across multiple tissues. The Human Protein Atlas (additional Web ref. 1) utilising mRNA expression data derived from RNA deep-sequencing studies of healthy tissues reports high expression levels of IGFBP6 in adipose tissue, endocrine and gastrointestinal systems, further confirmed by the report of The Genotype-Tissue Expression (GTEx) portal (additional Web ref. 2). All tendon samples analysed in the present study were dissected from the tendon midportion, limiting the possibility of including adipose or loose vascular connective tissue located in the paratenon. IGFBP6 cannot be considered as a tendon-specific gene, however, the expression difference between tendon and other musculoskeletal tissues and between different tendon phenotypes might be relevant in the context of tendon tissue engineering and detecting fibrocartilaginous degeneration.

Brianne Connizzo: How much of the conclusions do you think could be driven by variability in the data? Or what does the higher variability in tendon as compared to other tissues indicate about expression of these genes?
Authors: Target genes were chosen based on expression across two microarray platforms that were highly correlated for tendon (that was the selection criteria). Relative expression of many of these genes in cartilage and muscle is very low and should not be confused with 'very consistent'. Expression of certain genes in tendon may vary with site, age, injury and what seen may reflect the variation in the population. However, answering such a question was beyond the scope of the study due to the limited sample size and demographic characterisation of the investigated tissues.

Brianne Connizzo: How does expression of IGFBP6 change in injured or diseased tendon? Can this be used to identify varying levels of injury?

Authors: Previous studies report decreased expression of IGFBP6 in animal models of tendon injury and human fibrotic disease of the palmar fascia (Dupuytren's disease) (see Discussion). Timeseries study utilising collagenase-induced lesions of equine SDFT showed that IGFBP6 expression declines in response to the insult, however, it returns to pre-injury level within three months of injury (Dahlgren et al., 2006). That may suggest that tracking IGFBP6 expression change could indicate healing progress in acute tendon injuries if those finding would have been validated in naturally occurring disease. Preliminary experiment (data not published) demonstrated a significant decrease in IGFBP6 expression in early but not advanced rotator cuff tendinopathy as compared to healthy hamstring tendon. Those promising results warrant further investigation of IGFBP 6 expression in tendon health and disease. However, discussing IGFBP 6 role in tendon pathology was beyond the scope of the study as results described gene expression only in healthy tissue samples.

\section{Additional Web References}

1. https://www.proteinatlas.org [28.06.2019]

2. https://www.gtexportal.org/home/gene/IGFBP6 [19.07.2019]

Editor's note: The Scientific Editor responsible for this paper was Juerg Gasser. 